\title{
Modeling the radar scatter off of high-energy neutrino-induced particle cascades in ice
}

\author{
Krijn D. de Vries ${ }^{1, \star}$, Nick van Eijndhoven ${ }^{1}$, Aongus O’Murchadha ${ }^{2}$, Simona Toscano ${ }^{1}$, and Olaf \\ Scholten ${ }^{1,3}$ \\ ${ }^{1}$ Vrije Universiteit Brussel, Dienst ELEM, IIHE, Pleinlaan 2, 1050 Brussels, Belgium \\ ${ }^{2}$ UW Wisconsin, Madison, Wisconsin \\ ${ }^{3}$ University of Groningen, KVI-Center for Advanced Radiation Technology, 9747 AA Groningen, The Nether- \\ lands
}

\begin{abstract}
We discuss the radar detection method as a probe for high-energy neutrino induced particle cascades in ice. In a previous work we showed that the radar detection techniqe is a promising method to probe the high-energy cosmic neutrino flux above $\mathrm{PeV}$ energies. This was done by considering a simplified cascade geometry and scattering model. In this article we discuss the scattering in more detail. We provide a model for the radar cross-section based on the induced plasma properties, and discuss the angular dependence of the scatter.
\end{abstract}

\section{Introduction}

In [1], we discussed the radar detection technique as a probe for high-energy neutrino-induced particle cascades in ice. It was shown that if one is able to scatter efficiently, this technique should allow for the cost-efficient detection of neutrino-induced particle cascades above a few PeV. It follows that the radar detection method is a promising technique to cover the currently existing sensitivity gap between a few PeV where the IceCube neutrino observatory runs out of statistics [2], and a few EeV where the currently operating Askaryan radio detectors start to gain sensitivity [3, 4].

The main scatter occurs off of the over-dense ionization plasma left behind when a neutrino induced cascade traverses through the medium. To model the effective scattering cross-section, in [1], several approximations were made. The average free charge density within a tube of $2 \mathrm{~cm}$ radius was considered to estimate the plasma frequency. The scattering cross-section itself was consequently obtained from an emperically derived equation [5, 6]. Furthermore, skin-effects were ignored where the scatter was assumed to be isotropic.

In this article, we consider a more detailed model for the radar cross-section. Assuming radial symmetry, a two-dimensional model of the ionization plasma is given. Having the detailed free charge distribution, the scattering efficiency for layers of equal density will be considered by taking into account for the skin effect. Furthermore, the scattering itself is discussed where it is argued that instead of isotropic scattering a slit-like interference pattern can be expected.

\footnotetext{
^e-mail: krijndevries@gmail.com
} 


\section{The plasma}

Following [1], the starting point for the plasma model is the high-energy particle cascade which develops inside the ice. The electromagnetic cascade development as a function of depth $X\left(\mathrm{~g} / \mathrm{cm}^{2}\right)$ can be parameterized following [7, 8],

$$
N(X)=\frac{0.31 \exp \left[\left(X / X_{0}\right)(1-1.5 \ln s)\right]}{\sqrt{\ln \left(E / E_{\text {crit }}\right)}},
$$

where the radial particle distribution can be parameterized following [9],

$$
w(r)=\frac{\Gamma(4.5-s)}{\Gamma(s) \Gamma(4.5-2 s)}\left(\frac{r}{r_{0}}\right)^{s-1}\left(\frac{r}{r_{0}}+1\right)^{s-4.5} .
$$

Here $X_{0}=36.08 \mathrm{~g} / \mathrm{cm}^{2}$ denotes the electron interaction depth, $E_{\text {crit }}=0.0786 \mathrm{GeV}$ gives the critical energy for electrons in ice, and $E$ denotes the energy of the primary cascade-inducing particle. Additionally the shower age is given by $s(X)$, where $s=1$ denotes the shower age at shower maximum. The radial distance from the shower axis is given by $r$, where $r_{0}=7 \mathrm{~cm}$ for ice. For more detailes we would like to refer to Appendix A of [1].

The energy deposit from the high-energy cascade by ionization of the medium is large. Typically $90 \%$ of the cascade energy is finally deposited by ionizing the medium. The ionization profile is modeled considering an ionization loss of $2 \mathrm{MeV} / \mathrm{g} / \mathrm{cm}^{2}$ for a minimum ionizing particle. The ionization loss scales logarithmically with the energy of the ionizing particle and is therefore considered to be constant for the high-energy particles in the cascade front. Numerically it can easily be checked that the total energy loss due to ionization given by, $E_{\text {loss }}^{\text {ion }}=\int N(X) E_{\text {ion }} d X$, leads to an energy deposit of roughly $90 \%$. The ionization charge density is consequently obtained by assuming an ionization energy for ice equal to $E_{i o n}^{i c e}=20 \mathrm{eV}$.

\section{Over-dense scattering}

For over-dense scattering to occur, we need to fulfill the condition that the detection frequency is below the plasma frequency $v_{d}<v_{p}$. The plasma frequency on itself directly depends on the free charge density and mass, $v_{p}=8980 \sqrt{\frac{m_{e} n_{e}}{m_{p}}}$ Hz. Here $m_{e}$ denotes the electron mass, $m_{p}$ denotes the effective mass of the plasma constituent, and $n_{e}$ gives the free charge density. Following [10, 11], two types of plasma can be expected. A rather short lived free electron plasma, and a longer lived free proton plasma. In Fig. (1), we show the two dimensional plasma density profile for a $1 \mathrm{PeV}$ particle cascade. The over-dense region for the a proton-like plasma probed at $v_{d}=50 \mathrm{MHz}$ is indicated by the (white) contour line. It follows that already at $1 \mathrm{PeV}$ energies an over-dense tube with $\mathrm{O}(\mathrm{cm})$ radius and a longitudinal extension of $\mathrm{O}(\mathrm{m})$ can be expected for a proton-like plasma with $\mathrm{O}(\mu s)$ lifetime. The longitudinal extension of the plasma is given by either the plasma lifetime, $c \tau_{p}$, or the length of the cascade itself.

Having the density profile, we can now calculate the scattering cross-section. To illustrate the calculation, in this article we consider a simplfied geometry for which the incoming wave is normal, and the polarization is aligned, to the casacade direction. The scattering efficiency is accordingly obtained by considering (over-dense) layers of approximate equal density. Defining the skin depth $\delta_{p}=c / 2 v_{p}$, to be the depth in which the intensity falls by $e^{-1}$, the fraction of the signal absorbed and re-scattered in a single layer $i$ is given by,

$$
f_{\text {scatt }}^{i}=\left(1-f_{\text {scatt }}^{i-1}\right)\left(1-e^{-(\Delta x)^{i} / \delta_{p}^{i}}\right) .
$$




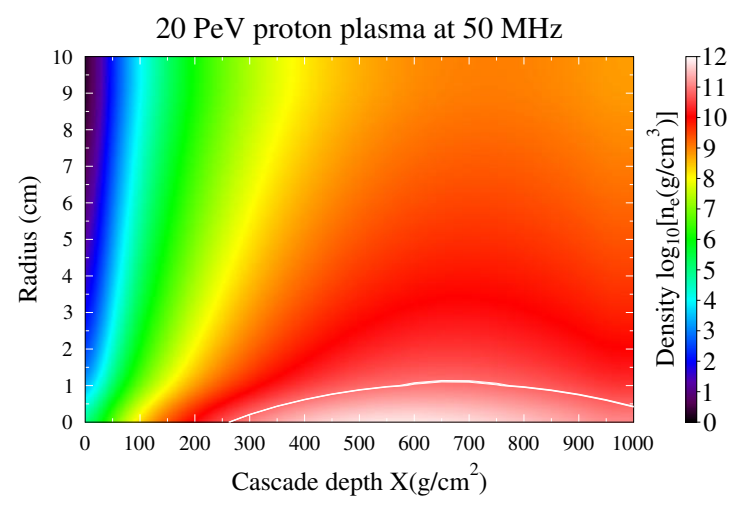

Figure 1. The two-dimensional plasma density profile for a $1 \mathrm{PeV}$ neutrino-induced particle cascade in ice. The over-dense proton-like plasma region probed at $v_{d}=50 \mathrm{MHz}$ is indicated by the (white) contour.

Here the factor $\left(1-f_{\text {scatt }}^{i-1}\right)$ takes into account the part of the signal which has already scattered, $(\Delta x)_{i}$ denotes the thickness of layer $i$, and $\delta_{p}^{i}$ denotes the plasma frequency in the considered layer. The total fraction of the incoming wave intensity which is scattered can now be obtained by,

$$
f_{\text {scatt }}=\sum_{i} f_{\text {scatt }}^{i}=\sum_{i}\left(1-f_{\text {scatt }}^{i-1}\right)\left(1-e^{-(\Delta x)^{i} / \delta_{p}^{i}}\right)
$$

For an incoming wave with normal incidence and its polarization aligned with the plasma, the scattering cross section is given by a folding with the geometrical area $A_{i}$ of layer $i$. This is a sigar-like surface, for which its area is estimated by, $A_{i}=L_{i} r_{i}^{\max } / 2$. The effective over-dense scattering cross section for a wave of normal incidence with its polarization aligned to the cascade and taking into account for skin-effects is thus given by,

$$
\sigma_{\text {od }}^{\text {norm }}=\sum_{i} f_{\text {scatt }}^{i} A_{i}=\sum_{i} A_{i}\left(1-f_{\text {scatt }}^{i-1}\right)\left(1-e^{-(\Delta x)^{i} / \delta_{p}^{i}}\right) .
$$

For the general case, a geometry factor has to be included to take into account the angle with respect to normal incidence and the polarization angle. Furthermore, it should be noted that there are still several unknowns that should be included into the effective radar cross section. So-far we did not consider the free charge collision rate, as well as the detailed plasma lifetime. These quantities are hard to model. Therefore, to determine the radar cross section the scattering efficiency has to be obtained experimentally. Initial experimental work has been performed during a beam-test experiment early 2015, where a first hint for a radar scatter was observed [12]. In the near future, a follow-up experiment is planned to confirm this hint.

\section{Diffraction}

Where in [1], the scattering was assumed to occur isotropically, in reality this only holds in the limit where the wavelength of the incoming wave is much larger than the scattering surface, $\lambda_{s c}>>A_{\text {scatt }}$. The other limiting situation is given when $\lambda_{s c}<<A_{\text {scatt }}$, for which specular reflection is expected. For efficient scattering, the detection frequency has to be below the plasma frequency, but still large 
enough to make multiple oscillations within the plasma lifetime which is expected to be in the nanosecond to microsecond range for the ionization plasma considered in this article. This immediately fixes the possible detection regime to $\mathrm{MHz}-\mathrm{GHz}$ frequencies.

For scattering off the plasma, we have to consider two length scales. The radial extension $r$ is small compared to the detection wavelength up to $\mathrm{GHz}$ frequencies, where the longitudinal extension $L$ is of the order of the detection wavelength. Hence, in this situation $\lambda_{s c} \approx A_{\text {scatt }}$, and we expect a diffraction pattern which can be approximated by Fraunhofer single slit diffraction. The intensity of the reflected signal therefore becomes an angular dependence given by,

$$
f_{\text {diff }}=\frac{I(\alpha)}{<I(\alpha)>}
$$

with,

$$
I(\alpha)=\operatorname{sinc}^{2}(\beta)
$$

where $\beta=(\pi L / \lambda) \sin \alpha$.

\section{Outlook}

In this article we consider a more realistic scattering model for the radar reflection off the ionization plasma due to a high-energy neutrino-induced particle cascade in ice. We describe a method to determine the over-dense radar cross-section for the ionization plasma. This method takes into account the skin-effect and is based on the two-dimensional plasma density profile. Furthermore, the emission pattern for a scattered signal is discussed. It follows that this pattern can be approximated by a single slit diffraction pattern. These considerations can be generalized to general scattering geometries, which should allow us to perform a detailed calculation for the radar scattering. The final goal of such a modeling effort is to calculate the sensitivity for a possible future high-energy neutrino detector based on the radar detection technique.

\section{References}

[1] K.D. de Vries, K. Hanson, T. Meures, Astropart. Phys. 60, 25-31 (2015)

[2] IceCube Collaboration, Science 342, 1242856 (2013)

[3] P. Allison et al., ARA Collaboration, Astropart. Phys. 35, 457-477 (2012)

[4] A. Nelles et al., ARIANNA Collaboration, these proceedings

[5] P.W. Gorham, Astropart. Phys. 15, 177 (2001)

[6] J.W. Crispin Jr., A.L. Maffett, Proc. Roy. Soc. A 53, 833 (1965)

[7] K. Kamata, J. Nishimura, Suppl. Progr. Theoret. Phys. 6, 93 (1958)

[8] K. Greisen, in: J.G. Wilson (Ed.), Prog. Cosmic Ray Phys., vol. III, North Holland, Amsterdam, 1965, p. 1

[9] K. Werner, K.D. de Vries, O. Scholten, Astroparticle Physics 37, 5-16 (2012)

[10] J.B. Verberne et al., Nature 272, 343-344 (1978)

[11] M. Kunst, J.M. Warman, Nature 288, 465-467 (1980)

[12] K.D. de Vries et al., PoS ICRC2015, 1168 (2015) 